\title{
Changes in left ventricular function and wall thickness in heart transplant recipients and their relation to acute rejection: an assessment by digitised $M$ mode echocardiography
}

H F J Mannaerts, A H M M Balk, M L Simoons, J Tijssen, S G van der Borden, P Zondervan, G R Sutherland, J R T C Roelandt

\begin{abstract}
Objective-Assessment of changes in left ventricular diastolic function and wall thickness after heart transplantation to verify whether these changes predicted acute rejection assessed by endomyocardial biopsy.

Design-Follow up according to a predefined protocol of consecutive patients from the first week after transplantation.
\end{abstract}

Setting-Heart transplantation unit of the Thoraxcentre, University Hospital Rotterdam Dijkzigt, The Netherlands.

Patients-All 32 patients undergoing orthotopic heart transplantation from 1 January 1989 to 31 March 1990 were examined. Two were excluded from the analysis. Patients were treated with cyclosporin and low dose steroids.

Main outcome measures-Data obtained by digitised $M$ mode echocardiography were compared with the results of endomyocardial biopsy (Billingham classification). Mean values for left ventricular wall thickness, internal dimension, and their standardised rates of change and fractional shortening were determined from 4-6 consecutive expiratory beats. Mean values and individual trends during follow up were also investigated for each ultrasound variable. The results of these average values were compared with values in a group of 10 healthy volunteers.

Results-Median follow-up was 177 days (range 10-399). Two hundred and sixty three consecutive $M$ mode studies were examined in relation to concurrent biopsy results. No significant differences were observed between the ultrasound variables at the time of moderate acute rejection (Billingham class $2, \mathbf{n}=37$ ) and other biopsy classes $(n=226)$. Nor did changes in individual patients predict (moderate) acute rejection episodes. Twenty six of the 30 patients had an abnormal (slow) left ventricular relaxation pattern throughout follow up.

Conclusions-Digitised left ventricular $M$ mode echocardiography did not predict the presence of acute rejec- tion. In most patients there was a persistent slow left ventricular relaxation pattern.

(Br Heart J 1992;68:356-64)

Systolic and diastolic left ventricular function and wall thickness in patients after heart transplantation may be altered by perioperative ischaemia and subsequent reperfusion damage, by acute and chronic rejection, and by postoperative systemic hypertension. Early studies suggested that increases in wall thickness or left ventricular mass shown by digitised $M$ mode echocardiography are moderately reliable markers of acute rejection in recipients of orthotopic heart transplants treated with azathioprine and prednisone immunosuppression..$^{1-2}$ In another study digitised $M$ mode echocardiography showed deterioration of diastolic left ventricular function in similarly treated patients. ${ }^{3}$ The clinical and haemodynamic signs of acute rejecton differ considerably in patients treated with cyclosporin/prednisone and those treated with azathioprine/prednisone. ${ }^{4}$ With cyclosporin/prednisone the onset of acute rejection is more insidious with less pronounced functional and structural changes in the heart during early stages of acute rejection. ${ }^{45}$ In two recent studies significant increases in left ventricular wall thickness (septum and posterior wall) and calculated left ventricular mass were found during moderate acute rejection (sensitivity $61 \%$ and $69 \%$ ) in patients treated with cyclospin/prednisone.$^{67}$ In three other studies, however, no increase in wall thickness or deterioration of systolic function (peak shortening rate of the left ventricular internal dimension) was seen during moderate acute rejection in similarly treated patients. ${ }^{8-10}$ Because of these conflicting results we prospectively studied digitised left ventricular $M$ mode echocardiograms in patients between one week and six months after transplantation.

Patients and methods

All 32 consecutive patients undergoing orthotopic heart transplantation during 1989 were 
enrolled into an observational unrandomised prospective study between January 1989 and March 1990 with serial follow up of consecutive patients according to a predefined protocol. For the detection of acute rejection by $M$ mode echocardiography each patient served as his own control and was followed up systematically from the first week after transplantation. Maintenance immunosuppressive treatment was cyclosporin and low dose prednisone. All patients were in sinus rhythm. Endomyocardial biopsy specimens for routine monitoring of acute rejection were taken at least 15 times in the first year according to a predefined protocol. Biopsy specimens were graded according to the Billingham classification. ${ }^{511}$ Biopsy specimens were taken every week for the first six weeks after heart transplantation. Thereafter they were taken during weeks $8,10,14,18,24,30,38,46,52$. Extra biopsies were performed in between these times if there was a clinical suspicion of acute rejection. In cases of moderate acute rejection (Billingham class 2) anti-rejection treatment was given (intravenous methylprednisolone or rabbit anti-thymocyte globulin). The globulin was given when moderate acute rejection occurred in the first two months after transplantation or when there was continuing moderate acute rejection despite previous anti-rejection treatment. In all other cases intravenous methylprednisolone was given. A biopsy was performed one week after the end of antirejection treatment.

Ultrasound recordings were obtained within six hours of biopsy. M mode tracings were recorded and analysed by one observer without prior knowledge of the biopsy results. Simultaneous cross sectional echocardiograms and left ventricular $M$ mode studies were obtained from the parasternal long axis view with either a 3.75 or $2.25 \mathrm{MHz}$ focused precordial transducer (Toshiba SSH-160 A). The patients were in the supine left lateral position with the transducer placed in the second to fourth left intercostal spaces. Care was taken to direct the $M$ mode cursor perpendicularly to the left ventricular long axis at the level of the chordal attachments to the mitral valve. Tracings were recorded at a paper speed of $100 \mathrm{~mm} / \mathrm{s}$ in combination with an electrocardiogram, phonocardiogram, and a respiratory tracing (based on changes in thoracic impedance). End diastole was taken as the onset of the first heart sound (mitral valve closure) and this was confirmed by the end of the mitral flow signal on a concurrent pulsed wave transmitral Doppler study. End systole was derived from the onset of the second heart sound on the phonocardiogram (A2, verified by recording an $M$ mode echocardiogram of aortic valve closure). Figure 1 shows an example of the characteristic left ventricular $\mathbf{M}$ mode recording obtained during the study with its corresponding digitised tracing. Off-line, 4-6 consecutive end expiratory beats were analysed with a software program developed in our laboratory. Similar analysis programmes were used by others. ${ }^{312}$ Care was taken to avoid artefacts in the $M$ mode measurements of the septum and posterior wall caused by chordae, papillary muscle, or pericardial effusion. This was done with close reference to videotaped simultaneous cross sectional echocardiograms. Wall thickness and internal dimensions were measured from leading edge to leading edge. The following variables were measured: IVSd and IVSs $=$ wall thickness of the interventricular septum at end diastole and end systole $(\mathrm{cm})$; LVPWd and LVPWs = wall thickness of the left ventricular posterior wall at end diastole and end systole (cm); TWT = total diastolic wall thickness (cm), which is the sum of IVSd + LVPWd; LVd and LVs = left ventricular internal dimension at end diastole and end systole $(\mathrm{cm})$; MWTCR = mean wall thickness cavity ratio, which is the sum of IVSd + LVPWd divided by two and again divided by LVd (synonym: wall thickness index); VCF = peak shortening rate of the left ventricular internal dimension $(\mathrm{cm} / \mathrm{s}) ; \mathrm{LVSR}=$ peak shortening rate of the left ventricular internal dimension and LVLR $=$ peak lengthening rate of left ventricular internal dimension, both standardised for instantaneous left ventricular internal dimension $\left(\mathrm{s}^{-1}\right)$; and PWTkR = peak rate of left ventricular posterior wall thickening $\left(\mathrm{s}^{-1}\right)$ and PWTnR = peak rate of left ventricular posterior wall thinning $\left(\mathrm{s}^{-1}\right)$, both standardised for instantaneous left ventricular posterior wall thickness. ${ }^{312}$ In addition the percentage fractional shortening of the left ventricular diameter was calculated as $((\mathbf{L V d}-\mathbf{L V s}) / \mathrm{LVd}$ $\times 100)$, in which LVd and LVs are the end diastolic and end systolic left ventricular internal dimensions. From the QRS complexes the $R R$ interval and heart rate were calculated. Results for all variables are expressed as the mean value of the 4-6 end expiratory consecutive beats measured. Longitudinal follow up was assessed by measuring the differences in $M$ mode variables between two consecutive examinations of the same patient. Thus the mean values of the actual examination minus the mean values of the previous examination were related to the concurrent transition from one biopsy class to another (Billingham classification).

The trough concentration of plasma cyclosporin ( $\mathrm{ng} / \mathrm{ml}$ ) was measured by a specific monoclonal radioimmunoassay ${ }^{13}$ on the same day as the endomyocardial biopsy and echocardiographic examinations. Brachial cuff blood pressures ( $\mathrm{mm} \mathrm{Hg}$ ) were measured on the same day.

The controls were 10 healthy volunteers (seven men and three women, mean (SD) age $29.6(4.5)$ years) without any symptoms or signs of heart disease and with normal electrocardiograms.

Reproducibility was assessed by an intra and inter observer variation test for which 120 beats in $25 \mathrm{M}$ mode recordings of different patients were reanalysed by either the same or a different observer.

\section{DATA ANALYSIS}

Intra and inter observer variations were determined by linear regression. The random errors were calculated by dividing the standard devia- 
Figure 1 (A)Left ventricular $M$ mode echocardiogram at paperspeed $100 \mathrm{~mm} / \mathrm{s}$ PHONO,

phonocardiogram; IVS, interventricular septum. LV dimension, left ventricular internal dimension; RESP, respiratory curve (trough in expiration); $L V P W$, left ventricular posterior wall; $E C G$,

electrocardiogram; mv, mitral valve; S1, onset of the first heart sound

(mitral closure); S2, onset of the second heart sound (aortic closure).

Sometimes the onset of S1 was not clear in every beat (as in beat 2 and 4). In these cases the timing of mitral valve closure by transmitral pulsed wave doppler was calibrated with the QRS complex (in this case the nadir of the $S$ wave on the ECG). (B) Continuous plot of the digitised contours of the interventricular septum, the left ventricular minor axis dimension, and the left ventricular posterior wall. The rates of change of the two left ventricular indices were obtained by differentiation. The vertical lines coincide with the peak rates of change. PWTnR and PWTkR are the peak posterior wall thinning and thickening rates both standardised for instantaneous posterior wall thickness. LVSR and LVLR are the peak shortening and peak lengthening rates, respectively, of left ventricular internal dimension, both standardised for instantaneous left ventricular internal dimension.

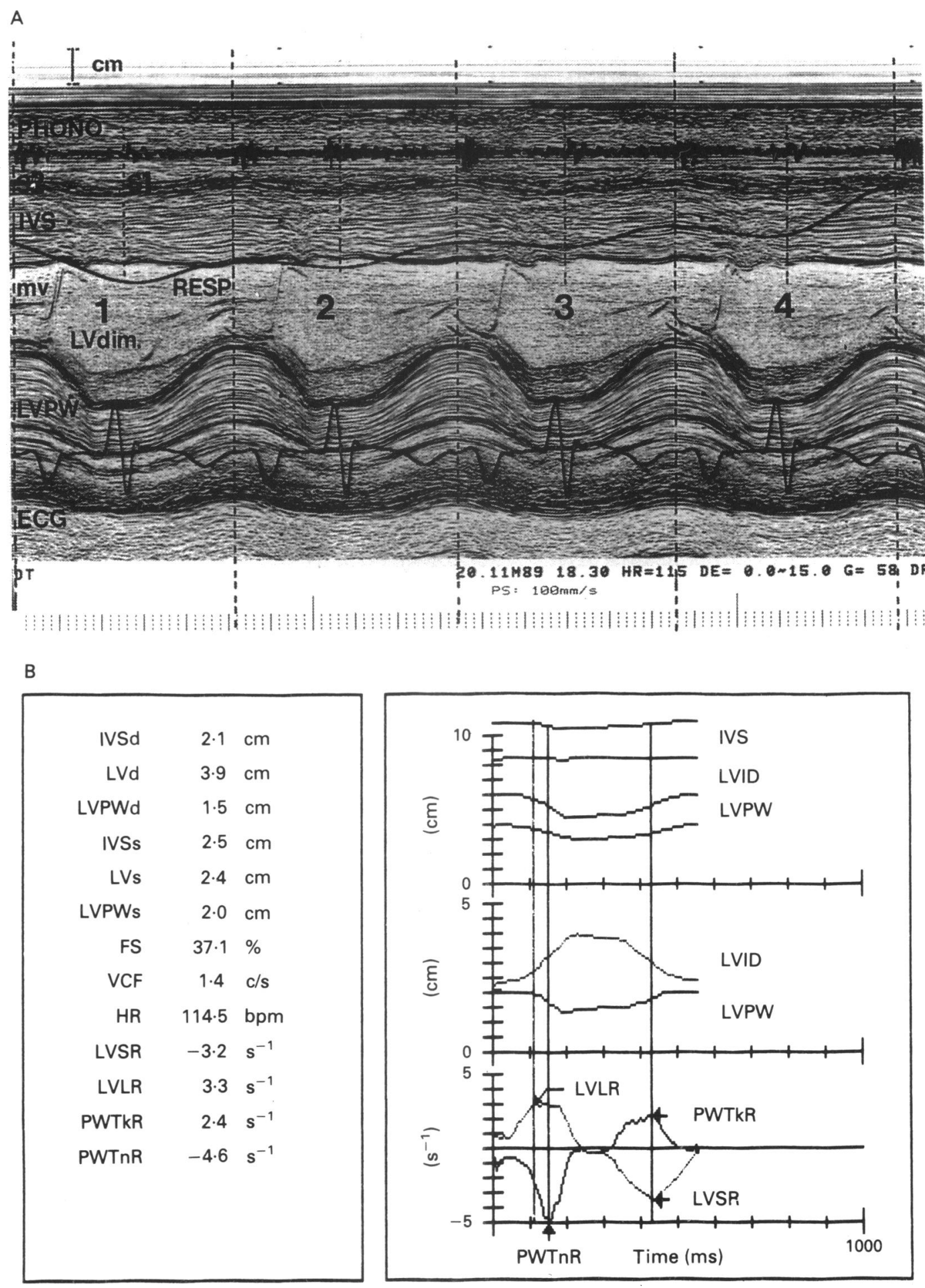

tion by the square root of the number of consecutive beats analysed $(\sqrt{ } 4)$. One way analysis of variance (Scheffé's test) was used to compare the mean values of $M$ mode variables or their individual differences, plasma cyclosporin concentrations, and blood pressures for the three biopsy classes. Linear regression was used to describe trends in time after cardiac transplantation for the $M$ mode varables studied. The derived individual slopes of the regression equations were tested for significance by a paired Student's $t$ test. Linear regression was also used to relate the wall thickness variables to left ventricular function. Similarly, duration of ischaemic time was related to various indices (averaged serial measurements in each patient). The MannWhitney $U$ test was used to test the averaged serial values of patients against values obtained in healthy volunteers.

\section{Results}

Table 1 shows the results for the intra and inter observer variation test. Two of the 32 patients were excluded from the analysis: one because no satisfactory $M$ mode readings could be obtained and the other because of prolonged septicaemia (two weeks), which is known to influence cardiac diastolic function. ${ }^{14}$ The 
Table 1 Intra and inter observer variation in patients after heart transplantation

\begin{tabular}{lll}
\hline & \multicolumn{2}{l}{ Variation $( \pm)$} \\
\cline { 2 - 3 }$M$ mode index & Intra & Inter \\
\hline HR $(/ \mathrm{min})$ & 0.50 & 0.55 \\
IVSd $(\mathrm{cm})$ & 0.04 & 0.04 \\
IVSs $(\mathrm{cm})$ & 0.08 & 0.08 \\
LVPWd $(\mathrm{cm})$ & 0.07 & 0.08 \\
LVPWs $(\mathrm{cm})$ & 0.09 & 0.09 \\
LVd $(\mathrm{cm})$ & 0.07 & 0.08 \\
LVs $(\mathrm{cm})$ & 0.09 & 0.10 \\
TWT $(\mathrm{cm})$ & 0.08 & 0.09 \\
MWTCR & 0.10 & 0.11 \\
FS $(\%)$ & 1.9 & 2.4 \\
VCF $(\mathrm{cm} / \mathrm{s})$ & 0.08 & 0.10 \\
PWTKR $\left(\mathrm{s}^{-1}\right)$ & 0.36 & 0.34 \\
PWTNR $\left(\mathrm{s}^{-1}\right)$ & 0.40 & 0.45 \\
LVSR $\left(\mathrm{s}^{-1}\right)$ & 0.26 & 0.29 \\
LVLR $\left(\mathrm{s}^{-1}\right)$ & 0.29 & 0.28 \\
\hline
\end{tabular}

Measurement variability analysed over 120 beats in 25 patients, expressed as random errors. See text for abbreviations.

remaining patient group consisted of 25 men and five women (mean (SD) age 43 (12) years). The duration of ischaemia for the donor heart (interval between cross clamping of the aorta in the donor and reperfusion in the recipient) was 161 (41) minutes. Two patients originally treated with cyclosporin/prednisone were given azathioprine from 28 and 40 weeks after cardiac transplantion, because they had several acute rejection episodes (Billingham class 2). Twenty nine of the 30 patients survived until the end of the follow up (median 177 days, range 10-399) after transplantation). One patient died 10 days after transplantation of severe acute rejection which was confirmed at necropsy. Only one $M$ mode recording had been obtained in this patient, a day before his death. At that time the biopsy specimen showed only mild acute rejection (Billingham class 1). The corresponding left ventricular $M$ mode echocardiogram showed that the internal dimension and wall thickness were normal, as was systolic function. Diastolic function, measured by peak posterior wall thinning rate was slightly abnormal.

At the end of the study a total of 263 biopsy results with concurrent $M$ mode recordings were available for analysis. The mean number of endomyocardial biopsy specimens and concurrent $M$ mode studies per patient was assessed for several intervals after transplantation. From 0-6 weeks this was 3.8; from $6-12$ weeks $1 \cdot 7$; from $12-26$ weeks $2 \cdot 3$; and after 26 weeks it was $1 \cdot 1$. The number of ultrasound examinations at these times were $113,50,68$, and 32 respectively. Overall $8.8 \mathrm{M}$ mode studies per patient were available for analysis. For each patient the serial measurements of left ventricular wall thickness and internal dimension and function during individual follow up were averaged. The means from the remaining 29 patients were pooled to give a group mean value (table 2 ). In patients the wall thickness of the interventricular septum and posterior wall and mean wall thickness to cavity ratio were considerably increased, whereas the left ventricular internal dimension was smaller than in the controls and in published reports. ${ }^{31516}$ Systolic function, as judged by the peak posterior wall thickening rate, was depressed but not as judged by left ventricular shortening rate or fractional shortening. The greatest abnormality, however, was seen in diastolic function. The peak posterior wall thinning rate was clearly abnormal in all but four patients (table 2 and fig 2). The peak rate of left ventricular lengthening was not significantly different from normal. Only four of 29 patients had a peak posterior wall thinning rate in the normal range for the healthy volunteers. For the remaining 25 the increased left ventricular wall thickness together with the decreased absolute values of peak posterior wall thinning rate were consistent with a slow relaxation associated with left ventricular hypertrophy. ${ }^{12}$ The latter is defined as IVSd $>1.2 \mathrm{~cm}$ and/or LVPWd $>1.1 \mathrm{~cm} .{ }^{15}$ According to this definition, and with some allowance for the measurement variability in our study (IVSd $>1.24 \mathrm{~cm}$ and LVPWd $>$ $1.17 \mathrm{~cm}$ ), hypertrophy of the septum was present in $101(38.4 \%)$ out of 263 records, and hypertrophy of the posterior wall in 191 $(72.6 \%)$ out of 263 records. The combination of both septal and posterior wall hypertrophy was seen in $85(32.3 \%)$ out of 263.

We analysed trends in time for all variables in 27 of the 29 patients. Two patients had to be excluded, because each had only two recordings. Linear regression analysis showed no trend in any of the variables studied. The relaxation abnormality persisted in 22 patients without a clear tendency towards improvement during follow up. In the remaining five there was a tendency towards improvement of peak posterior wall thinning rate (those with sig-

Table 2 Comparison of mean values in 30 patients during serial follow up and values for 10 controls

\begin{tabular}{|c|c|c|c|c|c|c|c|c|}
\hline & $L V d$ & $I V S d$ & $L V P W d$ & $F S$ & $L V S R$ & $P W T K R$ & $L V L R$ & $P W T N R$ \\
\hline \multicolumn{9}{|l|}{ Patients: } \\
\hline $\begin{array}{l}\text { Mean (SD) } \\
\text { Median } \\
\text { Range }\end{array}$ & $\begin{array}{l}4 \cdot 1(0 \cdot 3) \\
4 \cdot 1 \\
3 \cdot 3 \text { to } 4 \cdot 8\end{array}$ & $\begin{array}{l}1.2(0.2) \\
1.2 \\
0.9 \text { to } 1.7\end{array}$ & $\begin{array}{l}1.3(0.2) \\
1.3 \\
1.1 \text { to } 1.6\end{array}$ & $\begin{array}{l}37(7) \\
35 \\
26 \text { to } 55\end{array}$ & $\begin{array}{l}-2.8(0.7) \\
-2.7 \\
-5.0 \text { to }-1.9\end{array}$ & $\begin{array}{l}2.6(0.4) \\
2.5 \\
1.9 \text { to } 3.4\end{array}$ & $\begin{array}{l}3.6(0.8) \\
3.3 \\
2.5 \text { to } 5.7\end{array}$ & $\begin{array}{l}-3 \cdot 8(0 \cdot 8) \\
-3 \cdot 5 \\
-5 \cdot 7 \text { to }-2 \cdot 8\end{array}$ \\
\hline $\begin{array}{l}\text { Controls: } \\
\text { Mean (SD) } \\
\text { Median } \\
\text { Range }\end{array}$ & $\begin{array}{l}4 \cdot 8(0 \cdot 3) \\
4 \cdot 8 \\
4 \cdot 3 \text { to } 5 \cdot 1\end{array}$ & $\begin{array}{l}1 \cdot 0(0 \cdot 1) \\
1 \cdot 0 \\
0 \cdot 8 \text { to } 1 \cdot 1\end{array}$ & $\begin{array}{l}0.8(0.2) \\
0.8 \\
0.4 \text { to } 1.0\end{array}$ & $\begin{array}{l}37(3) \\
37 \\
30 \text { to } 43\end{array}$ & $\begin{array}{l}-2.4 \text { to } 0.4 \\
-3.4 \\
-2.4 \text { to }-4.1\end{array}$ & $\begin{array}{l}3.5(1.0) \\
3.3 \\
2.0 \text { to } 5.6\end{array}$ & $\begin{array}{l}3.3(0 \cdot 6) \\
3 \cdot 4 \\
2 \cdot 4 \text { to } 4 \cdot 1\end{array}$ & $\begin{array}{l}-7 \cdot 1(2.5) \\
-5.9 \\
-12.1 \text { to }-4.5\end{array}$ \\
\hline $\mathrm{p}^{\star}$ & $<10^{-4}$ & $<3 \cdot 10^{-3}$ & $<3 \cdot 10^{-6}$ & NS & NS & $<2 \cdot 10^{-3}$ & NS & $<3 \cdot 10^{-5}$ \\
\hline
\end{tabular}

LVd, end diastolic left ventricular internal dimension (cm); IVSd, end diastolic septal thickness (cm); LVPWd, end diastolic thickness of left ventricular posterior wall $(\mathrm{cm})$; FS, fractional shortening (\%); LVSR, peak left ventricular shortening rate; LVLR, peak left ventricular lengthening rate $\left(\mathrm{s}^{-1}\right)$; PWTKR, peak posterior wall thickening rate; PW'NR, peak posterior wall thinning rate $\left(s^{\prime}\right)$. All rates are standardised for instantaneous left ventricular internal dimension or posterior

$\star$ Patients $v$ controls. 

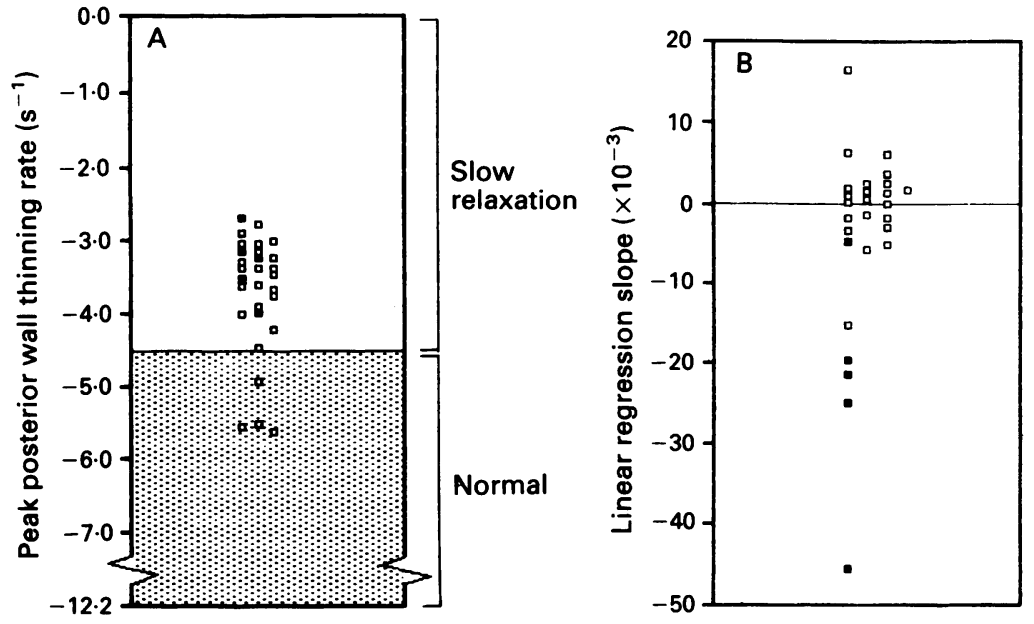

Figure 2 (A) Averaged serial data of peak posterior wall thinning rate for 29 patients. A patient who died and two others have been omitted. The normal range is indicated (range $-12 \cdot 2$ to $-4 \cdot 5 \mathrm{~s}^{-1}$ ). Twenty five patients had values outside the normal range and showed a slow left ventricular relaxation pattern. (B) Linear regression slopes of peak posterior wall thinning rate for 27 of the 29 patients. The group showed no significant trend for this index. Only five patients had significant negative slopes (black squares) and thus showed a tendency towards an improvement in their slow relaxation pattern. Regression slopes for the other patients were not statistically significant. ventricular wall thickness, internal dimension, and systolic and diastolic function grouped according to the concurrent biopsy class. In patients with biopsy class 0 , interventricular septal wall thickness (either at end diastole or end systole) was increased. In the pooled data for the other indices no significant differences were seen. Trough concentrations of plasma cyclosporin, heart rate (sinus tachycardia), and blood pressure were similar in all biopsy classes. Biopsy classes could not be discriminated on the basis of indices of systolic or diastolic function.

Table 4 shows the pooled data of individual changes in $\mathrm{M}$ mode variables, grouped according to transitions from one biopsy class to another (in total 234 transitions). For all ultrasound variables studied there were no significant differences between the pooled data of several clinically important transitions from one histological group to another. Therefore, this method did not allow diagnosis of moderate acute rejection either. The large standard deviations in tables 3 and 4 show that there is a wide overlap between biopsy classes. Figure 5 gives an example of an individual plot for various $M$ mode variables versus follow up time. In this patient the first moderate acute rejection episode did not coincide with an absolute low value of peak posterior wall thinning rate whereas the second one did. These low values for posterior wall thinning rate, however, were not specific for moderate acute rejection. Similarly, changes in the other $M$ mode indices during acute rejection episodes were not specific either. left ventricular relaxation. Figure 4 shows linear regression plot for the correlation between left ventricular posterior wall thickness (measured in diastole (LVPWd) and peak posterior wall thickening (PWTkR) and thinning rates (PWTnR).

Correlations between blood pressure measurements and $M$ mode indices of wall thickness and left ventricular function were generally poor. Therefore, instantaneous blood pressure did not exert an important influence on the results of this study.

Table 3 shows the ultrasound indices of left

\section{Figure 3 Relation}

between mean serial values of peak posterior wall thinning rate and the total duration of ischaemic time for each of the 29 patients. Linear regression model: $Y=a+b X ; a$ (intercept) $=-4.90 ; b$ (slope) $=0.0072$; correlation coefficient $=$ $0 \cdot 37$. Linear regression showed a significant trend for the absolute value of this index to decrease as the ischaemia time increased. The $95 \%$ confidence interval of the regresson line is shown.

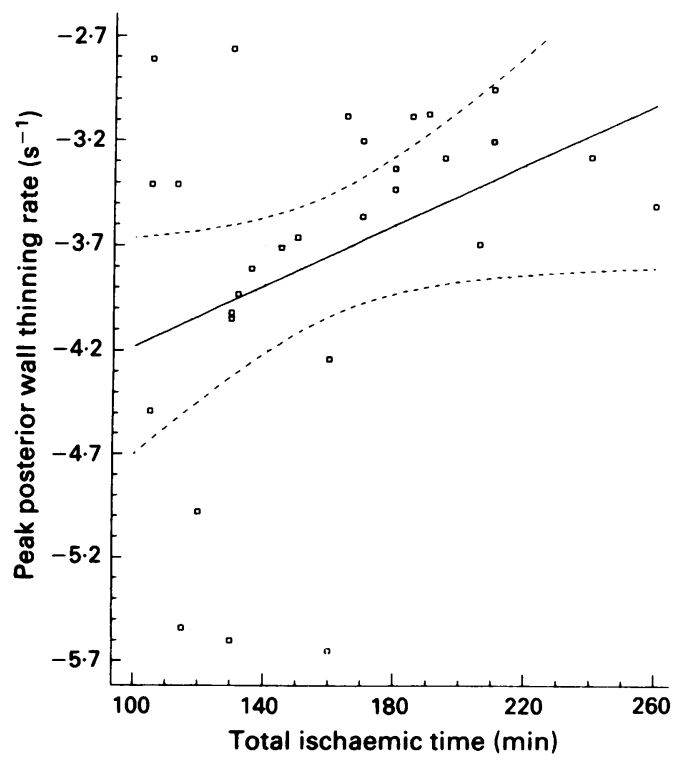

\section{Discussion}

The aim of this prospective study was to assess a possible trend with time in left ventricular systolic and diastolic function and wall thickness in recipients of orthotopic heart transplants and to verify whether changes related to rejection could be detected by ultrasound. Digitised left ventricular $\mathbf{M}$ mode echocardiography was used because it provides quantitative information on wall thickness, left ventricular dimension, and systolic and diastolic function, including rates of left ventricular posterior wall thinning and thickening. These variables have been reported to be sensitive markers of diastolic and systolic function. ${ }^{3} 1217$

REPRODUCIBILITY OF M MODE VARIABLES

The results indicate that the reproducibility of the variables left ventricular dimension and wall thickness (expressed as random errors) was good and that it was acceptable for the ratio of mean wall thickness to cavity and standardised rates of change. The latter are clearly influenced by the standardisation for instantaneous posterior wall thickness or instantaneous left ventricular internal dimension. Therefore the error in peak VCF is much smaller than that of peak left ventricular shortening rate, which is standardised to the instantaneous left ventricular internal dimension. 
Figure 4 Linear regression of $(A)$ peak posterior wall thinning rate (PWTnR) and $(B)$ peak posterior wall thickening rate (PWTkR on diastolic thickness of the left ventricular posterior wall ( $L V P W d$ ). The corresponding linear regression equations are indicated. The inner broken lines are the 95\% confidence intervals of the linear regression line and the outer broken lines are the $95 \%$ prediction limits for any given values of $P W T n R, P W T k R$, and LVPWd.
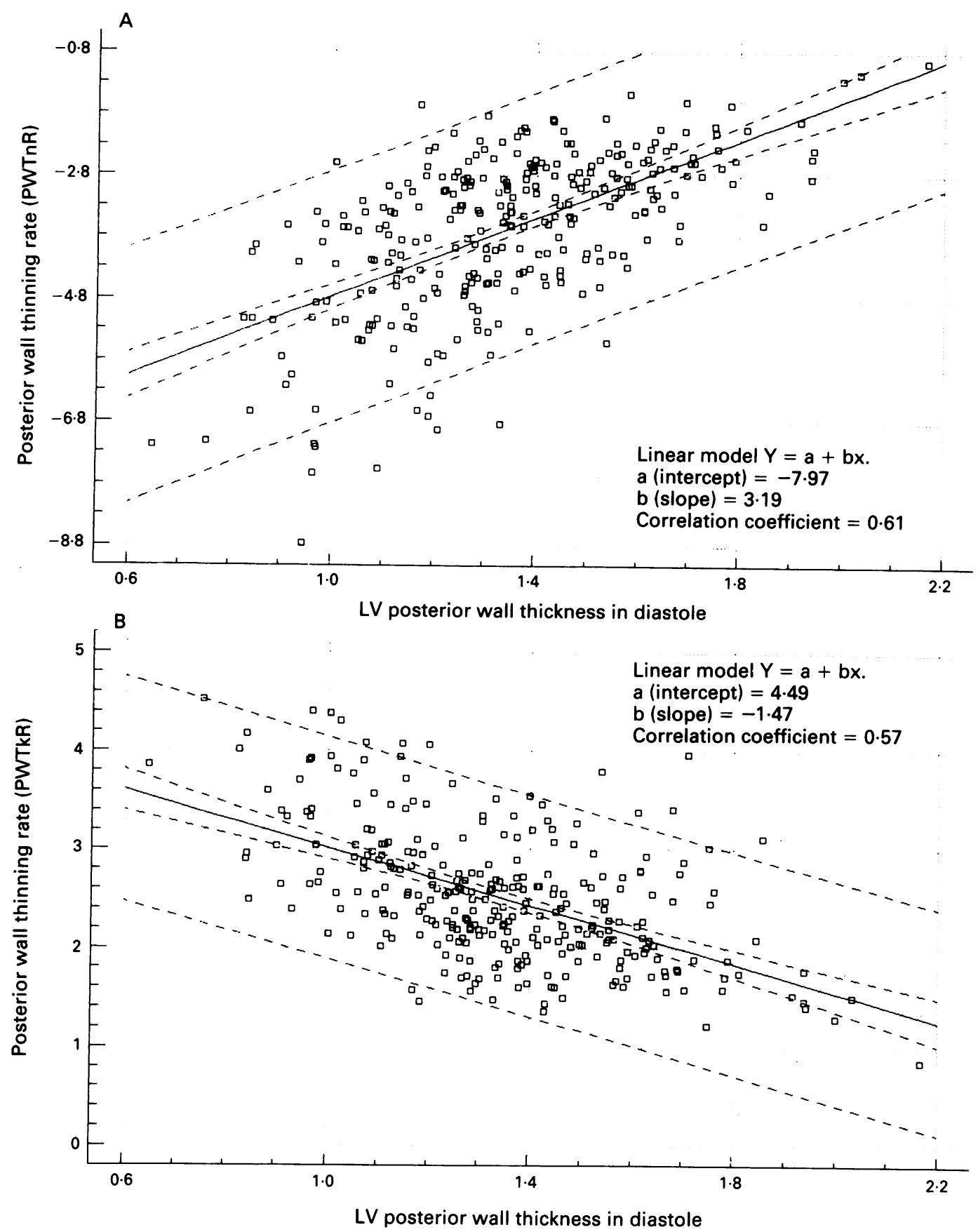

SLOW LEFT VENTRICULAR RELAXATION PATTERN Wall thickness was increased and both the left ventricular internal dimension and peak posterior wall thinning rate were decreased in all patients whether or not they showed evidence of acute rejection. This result indicates a slow left ventricular relaxation pattern associated with left ventricular hypertrophy that persisted during follow up in 22 out of 27 patients. These data were compared with another study of left ventricular digitised $M$ mode echocardiography in patients with non-obstructive hypertrophic cardiomyopathy in association with long-standing hypertension and in patients with cardiac amyloidosis. ${ }^{12}$ The left ventricular wall thickness and peak posterior wall thinning rates in our patients were typical of the group with hypertension. The data on left ventricular internal dimension, however, resembled those obtained in patients with restrictive car- diomyopathy caused by cardiac amyloidosis. Increased myocardial stiffness or signs of a restrictive physiology were reported by others using $M$ mode echocardiography and cardiac catheterisation, ${ }^{18}$ serial right heart catheterisation, ${ }^{19}$ or pulsed wave transmitral Doppler. ${ }^{20}$ This slow relaxation/restrictive filling pattern may be caused by fibrosis and persistent cellular infiltration and oedema (that is, mild acute rejection) within the myocardium or by left ventricular hypertrophy caused by systemic hypertension. All these factors tend to increase wall thickness and have a negative effect on both systolic and diastolic function, as illustrated by the inverse relation between the rates of diastolic wall thickness and posterior wall thinning and thickening (fig 4). Furthermore, impairment of the passive diastolic properties of the left ventricle was reported to correlate with the ischaemic time of the donor heart, even if 
Table 3 mode indices in various Billingham classes (mean (SD)

\begin{tabular}{|c|c|c|c|}
\hline & Class $0(n=49)^{\star}$ & Class $1(n=177)^{\star}$ & Class $2(n=37)^{\star}$ \\
\hline $\begin{array}{l}\text { Plasma cyclosporin }(\mathrm{ng} / \mathrm{ml}) \\
\text { Systolic blood pressure }(\mathrm{mm} \mathrm{Hg}) \\
\text { Diastolic blood pressure }(\mathrm{mm} \mathrm{Hg}) \\
\text { Heart rate (beats/min) }\end{array}$ & $\begin{array}{l}154(172) \\
144(21) \\
96(13) \\
92(13)\end{array}$ & $\begin{array}{r}130(66) \\
143(15) \\
95(11) \\
94(10)\end{array}$ & $\begin{array}{r}141(80) \\
141(16) \\
93(13) \\
97(12)\end{array}$ \\
\hline $\begin{array}{l}\text { End diastolic indices: } \\
\text { Mean wall thickness cavity ratio } \\
\text { Total wall thickness }(\mathrm{cm}) \\
\text { Interventricular septum }{ }^{\dagger}(\mathrm{cm}) \\
\text { Left ventricular internal dimension }(\mathrm{cm}) \\
\text { Left ventricular posterior wall }(\mathrm{cm})\end{array}$ & $\begin{array}{l}0 \cdot 3(0 \cdot 1) \\
2 \cdot 6(0 \cdot 4) \\
1 \cdot 3(0 \cdot 3) \\
4 \cdot 2(0 \cdot 5) \\
1 \cdot 3(0 \cdot 3)\end{array}$ & $\begin{array}{l}0 \cdot 3(0 \cdot 1) \\
2 \cdot 5(0 \cdot 4) \\
1 \cdot 2(0 \cdot 3) \\
4 \cdot 2(0 \cdot 4) \\
1 \cdot 3(0 \cdot 3)\end{array}$ & $\begin{array}{l}0 \cdot 3(0 \cdot 1) \\
2 \cdot 5(0 \cdot 4) \\
1 \cdot 2(0 \cdot 3) \\
4 \cdot 0(0 \cdot 4) \\
1 \cdot 3(0 \cdot 3)\end{array}$ \\
\hline $\begin{array}{l}\text { End systolic indices: } \\
\text { Interventricular septum } \dagger(\mathrm{cm}) \\
\text { Left ventricular internal dimension }(\mathrm{cm}) \\
\text { Left ventricular posterior wall }(\mathrm{cm})\end{array}$ & $\begin{array}{l}1.6(0.4) \\
2.5(0.5) \\
2.0(0.3)\end{array}$ & $\begin{array}{l}1.5(0.3) \\
2.7(0.5) \\
1.9(0.3)\end{array}$ & $\begin{array}{l}1.5(0.3) \\
2.6(0.4) \\
2.0(0.4)\end{array}$ \\
\hline $\begin{array}{l}\text { Indices of left ventricular function: } \\
\text { Fractional shortening }(\%) \\
\text { Peak VCF }(\mathrm{cm} / \mathrm{sec}) \\
\text { Peak left ventricular shortening rate }\left(\mathrm{s}^{-1}\right) \\
\text { Peak left ventricular lengthening rate }\left(\mathrm{s}^{-1}\right) \\
\text { Peak posterior wall thickening rate }\left(\mathrm{s}^{-1}\right) \\
\text { Peak posterior wall thinning rate }\left(\mathrm{s}^{-1}\right)\end{array}$ & $\begin{array}{r}39 \cdot 4(9 \cdot 2) \\
1 \cdot 4(0 \cdot 3) \\
-3 \cdot 0(1 \cdot 1) \\
3 \cdot 8(1 \cdot 1) \\
2 \cdot 7(0 \cdot 7) \\
-3 \cdot 9(1 \cdot 4)\end{array}$ & $\begin{array}{r}36 \cdot 0(9 \cdot 0) \\
1 \cdot 3(0 \cdot 4) \\
-2 \cdot 7(1 \cdot 0) \\
3 \cdot 4(1 \cdot 0) \\
2 \cdot 5(0 \cdot 7) \\
-3 \cdot 6(1 \cdot 3)\end{array}$ & $\begin{array}{r}36 \cdot 5(9 \cdot 3) \\
1 \cdot 3(0 \cdot 4) \\
-2 \cdot 8(0 \cdot 9) \\
3 \cdot 5(1 \cdot 0) \\
2 \cdot 6(0 \cdot 7) \\
-3 \cdot 8(1 \cdot 3)\end{array}$ \\
\hline
\end{tabular}

^Number of echocardiographic examinations.

tp $<0.05$ for differences between classes 0 and 1 for both end diastolic and end systolic thickness of the interventricular septum.

assessed one year after orthotopic heart transplantation. ${ }^{18}$ This accords with our findings (fig 3).

\section{DETECTION OF MODERATE ACUTE REJECTION}

In the present study we detected no significant differences in left ventricular digitised $\mathrm{M}$ mode variables between moderate acute rejecton and its absence, either at group level or in individual patients. In this respect our data are consistent with three other studies in similarly treated patients. ${ }^{8-10}$ The observed differences at group level between Billingham class 0 (no rejection) and class 1 (mild rejection) in both systolic and diastolic interventricular septal wall thickness are considered to be of no clinical importance and in individual patients did not reach -significance when measured serially. Class 0 biopsy specimens were more common in the early postoperative period and the increased wall thickness in this biopsy class may be related to myocardial oedema caused by ischaemia and subsequent reperfusion injury. In contrast to other studies in cyclosporin treated patients ${ }^{67}$ the calculation of total wall thickness (TWT) did not give better results than the measurement of either interven- tricular septal or posterior wall thickness. The explanation for this difference in results between our study and the studies mentioned for the detection of moderate acute rejection by measurement of total wall thickness may lie in left ventricular hypertrophy (especially of the posterior wall) and/or persisting cellular infiltration within the myocardium (Billingham class 1 ). In contrast to the two other studies both these factors were present in most of our patients. Increases in wall thickness caused by moderate acute rejection are harder to detect in the presence of these factors. We do not know whether the absence of left ventricular hypertrophy in the other two studies could be attributed to lower plasma concentrations of cyclosporin.

The considerable overlap of digitised $M$ mode variables between biopsy classes and the fluctuations during individual follow up might be explained by the continuous interplay of changes in haemodynamic factors other than those associated with acute rejection, such as pulmonary hypertension; ischaemic injury and subsequent reperfusion injury with its associated oedema, which gradually diminish after transplantation; and the onset of hyper-

Table 4 Transition from one biopsy class to another with concurrent changes in digitised $M$ mode indices

\begin{tabular}{|c|c|c|c|c|c|c|c|c|c|}
\hline & \multicolumn{9}{|c|}{ Billingham class } \\
\hline & $0 \rightarrow 1$ & $0 \rightarrow 2$ & $1 \rightarrow 2$ & $1 \rightarrow 0$ & $2 \rightarrow 1$ & $2 \rightarrow 0$ & $0 \rightarrow 0$ & $1 \rightarrow 1$ & $2 \rightarrow 2$ \\
\hline $\mathrm{N}$ & 25 & 5 & 27 & 24 & 30 & 2 & 13 & 104 & 4 \\
\hline $\begin{array}{l}\text { HR } \\
\text { IVSd } \\
\text { IVSs } \\
\text { LVPWd } \\
\text { LVPWs } \\
\text { LVd } \\
\text { LVs } \\
\text { TWT } \\
\text { MWTCR } \\
\text { FS } \\
\text { VCF } \\
\text { PWTKR } \\
\text { PWTNR } \\
\text { LVSR } \\
\text { LVLR }\end{array}$ & $\begin{array}{l}-0.1(10) \\
-0.02(0.18) \\
-0.01(0.29) \\
0(0.26) \\
-0.04(0.22) \\
-0.05(0.39) \\
0.10(0.33) \\
-0.01(0.32) \\
-0.00(0.05) \\
-3.1(8.0) \\
-0.11(0.28) \\
-0.14(0.85) \\
0.36(1.44) \\
0.16(0.74) \\
-0.32(0.94)\end{array}$ & $\begin{array}{r}-0.3(11) \\
-0.05(0.13) \\
0.04(0.16) \\
-0.11(0.25) \\
-0.04(0.20) \\
0.02(0.46) \\
0.06(0.26) \\
-0.24(0.27) \\
-0.04(0.01) \\
-1.2(2.0) \\
-0.13(0.10) \\
0.12(0.55) \\
-0.58(2.00) \\
0.28(0.20) \\
0.18(0.85)\end{array}$ & $\begin{array}{l}2.7(8) \\
0.02(0.23) \\
0.03(0.22) \\
0.01(0.27) \\
-0.03(0.21) \\
-0.09(0.28) \\
0.09(0.31) \\
0.01(0.39) \\
0.01(0.06) \\
-3.7(8.1) \\
-0.09(0.30) \\
0.12(0.87) \\
-0.15(1.42) \\
0.26(0.90) \\
-0.25(0.93)\end{array}$ & $\begin{array}{r}3.1(6) \\
0.01(0.23) \\
-0.01(0.25) \\
0.07(0.24) \\
0.10(0.30) \\
-0.04(0.42) \\
-0.09(0.43) \\
0.08(0.36) \\
0.01(0.05) \\
1.8(7.1) \\
0.04(0.28) \\
0.09(0.58) \\
0.06(1.19) \\
-0.21(0.66) \\
0.34(1.03)\end{array}$ & $\begin{array}{r}-0.6(8) \\
0.01(0.20) \\
-0.02(0.22) \\
-0.01(0.24) \\
-0.01(0.33) \\
0.01(0.34) \\
-0.09(0.33) \\
0.02(0.28) \\
0.00(0.05) \\
2.7(8.5) \\
0.10(0.36) \\
-0.10(0.87) \\
0.13(1.35) \\
0.23(1.09) \\
0.16(1.14)\end{array}$ & $\begin{array}{r}-1.6(2) \\
-0.21(0.42) \\
0.15(0.22) \\
-0.16(0.35) \\
-0.53(0.75) \\
0.29(0.55) \\
0.39(0.37) \\
-0.38(0.76) \\
-0.07(0.14) \\
-6.5(2.7) \\
-0.30(0.04) \\
-0.50(0.70) \\
0.69(1.03) \\
0.91(0.61) \\
-1.10(1.37)\end{array}$ & $\begin{array}{r}-2.5(7) \\
-0.06(0.33) \\
-0.04(0.35) \\
0.02(0.28) \\
0.04(0.05) \\
0.07(0.14) \\
-0.09(0.42) \\
-0.05(0.37) \\
-0.01(0.04) \\
3.1(10.2) \\
0.08(0.38) \\
-0.00(0.30) \\
-0.14(1.03) \\
-0.30(1.13) \\
0.18(1.22)\end{array}$ & $\begin{array}{r}0.6(7) \\
-0.02(0.19) \\
-0.01(0.23) \\
-0.02(0.28) \\
-0.02(0.29) \\
0.05(0.44) \\
0.03(0.45) \\
-0.03(0.35) \\
-0.01(0.06) \\
-0.2(8.1) \\
-0.02(0.32) \\
-0.02(0.83) \\
-0.12(1.40) \\
0.08(0.92) \\
0.02(1.06)\end{array}$ & $\begin{array}{l}-1.9(13) \\
-0.01(0.34) \\
0.16(0.22) \\
-0.02(0.21) \\
-0.03(0.27) \\
-0.07(0.24) \\
-0.35(0.41) \\
-0.03(0.47) \\
-0.00(0.07) \\
7.7(11.2) \\
0.23(0.35) \\
-0.08(0.58) \\
0.89(1.48) \\
-0.48(0.91) \\
0.50(1.27)\end{array}$ \\
\hline
\end{tabular}

$\mathrm{N}$, number of observations for transitions between biopsy classes. The mean differences (1 SD) are presented for several transitions. For abbreviations and units of measurement see text and other tables. 


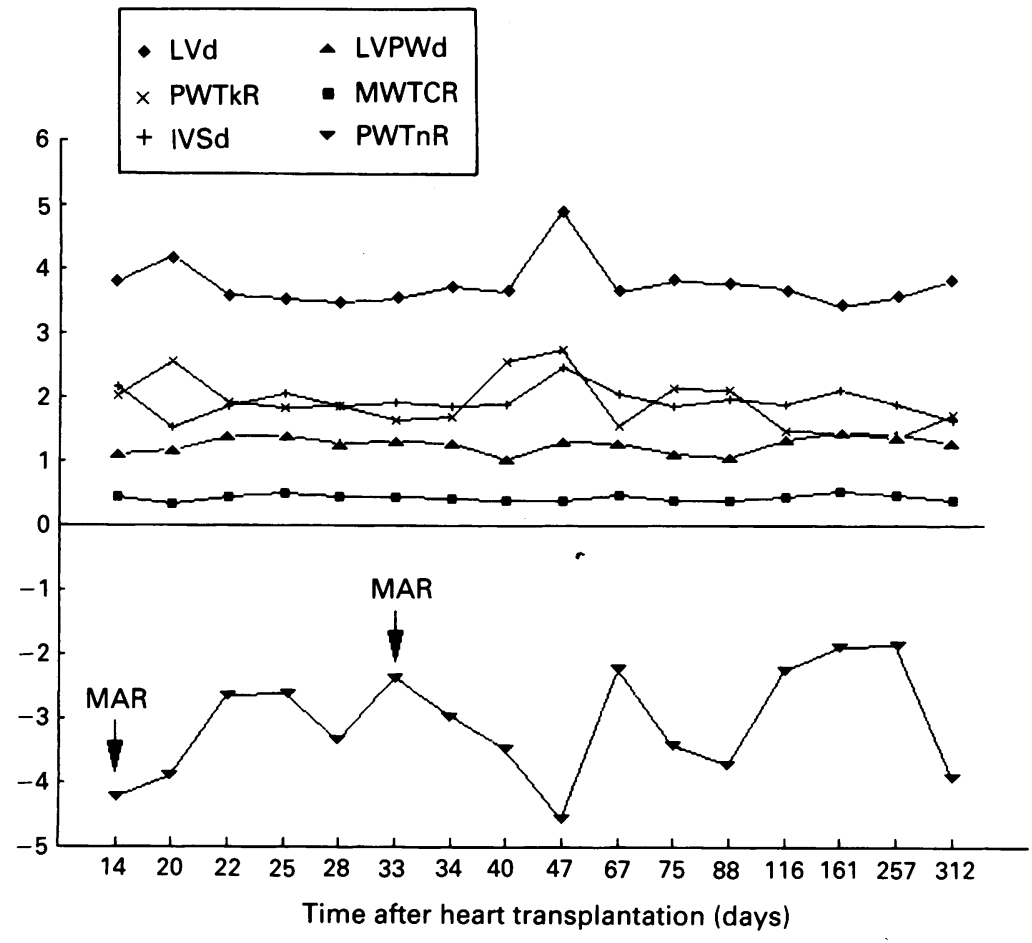

Figure 5 Trends in six left ventricular digitised $M$ mode indices in one patient during follow up (days after transplantation). LVd, diastolic thickness of the left ventricular internal dimension; PWTkR, peak posterior wall thickening rate; IVSd, diastolic thickness of the interventricular septum; LVPWd, diastolic thickness of the left ventricular posterior wall; MWTCR, mean wall thickness cavity ratio; PWTnR, peak posterior wall thinning rate; $M A R$, moderate acute rejection.

tension induced left ventricular hypertrophy. ${ }^{10}$ Finally, antihypertensive treatment, including diuretics, calcium antagonists, angiotensin converting enzyme inhibitors, other vasodilators, or sometimes $\beta$ blocking agents, has a profound effect on loading conditions and the inotropic and lusiotropic states. ${ }^{21}$

It is unlikely that the overlap of $M$ mode variables between biopsy classes is caused by false negative biopsy results because of sampling error, which is reported to be low (2 to $5 \%){ }^{22}$ The plasma cyclosporin concentration was not lower in the acute rejection groups (biopsy classes 1 and 2) and therefore myocardial wall thickness (as a marker of myocardial tissue reactivity during acute rejection) was not dependent on the cyclosporin concentration. In our hospital, however, biopsy specimens are classified as "moderate acute rejection" requiring rejection therapy only if an interstitial cellular infiltrate together with myocyte necrosis is detected. Fibrosis and interstitial oedema, which may affect ventricular function, are often present in biopsy specimens classified as "mild acute rejection". Therefore it is not surprising that $M$ mode indices of left ventricular systolic and diastolic function do not seem to be related to the histological classification.

\section{LIMITATIONS OF THE STUDY}

There were three. Firstly, we did not take account of the effects of recipient atrial contraction and relaxation, which may affect all parts of the cardiac cycle. ${ }^{23}$ The recipient's atrial $P$ waves on the electrocardiogram are objective criteria for the presence of recipient atrial contraction within the cardiac cycle. ${ }^{23}$ In our experience recipient atrial $\mathrm{P}$ waves can only be seen in about $18 \%$ of all tracings despite the presence of very clear donor $\mathbf{P}$ waves. This means that in about $82 \%$ of all tracings we were unable to use an objective criterion for the presence of recipient atrial contraction. Furthermore, in pulsed wave transmitral Doppler studies of diastolic left ventricular function beat to beat variability induced by respiration may be larger than the variability induced by recipient atrial contraction, especially in patients with pericardial effusion. ${ }^{2324}$ To our knowledge there are no reports of the variability of digitised $M$ mode indices but presumably the variability would be of a similar magnitude and direction as in pulsed wave transmitral Doppler studies. Because most of our patients had small to moderate pericardial effusions during follow up, respiratory induced variability must have been substantial. We reduced this major source of beat to beat variability by analysing 4 to 6 consecutive end expiratory beats.

Secondly, digitised left ventricular $M$ mode echocardiography in fact only assesses the function of the basal portion of the left ventricle. Some degree of paradoxical septal motion was present in most of the patients, and could have influenced the indices measured by digitised $M$ mode echocardiography although posterior wall dynamics are unlikely to have been affected by this phenomenon.

Thirdly, with left ventricular $M$ mode echocardiography there will be pseudo-hypokinesis of the septum and pseudo-hyperkinesis of the left ventricular posterior wall affecting posterior wall dynamics, peak left ventricular lengthening and shortening rates and fractional shortening. This is inherent in any one dimensional technique with a fixed reference point, and is caused by the anterior motion of the entire heart relative to the transducer. The progressive restraining effect of postoperative adhesions reduces this anterior motion of the entire heart after the first postoperative months. ${ }^{25}$ In the presence of such adhesions motion of the entire heart is mainly caused by respiration if there is no large pericardial effusion. We reduced this problem by predominantly studying end expiratory beats, in which there is little respiratory influence on motion of the entire heart.

In most patients with heart transplants there was a slow left ventricular relaxation pattern during follow up. In a subset of patients there was a tendency towards improvement of this pattern. Left ventricular hypertrophy caused by systemic hypertension, myocardial fibrosis, and persisting mild acute rejection are the most probable explanations for the slow left ventricular relaxation pattern. The total duration of ischaemic time of the donor heart was inversely related to the peak posterior wall thinning rate, and may have contributed to the slow left ventricular relaxation in patients with a long ischaemic time. Digitised left ventricular $M$ mode echocardiography did not allow the non-invasive diagnosis of moderate acute rejec- 
tion, either in a group or in an individual. This may be due to various haemodynamic factors that influence both wall thickness and cardiac function after cardiac transplantation.

This study was supported by grant $88 \cdot 110$ of the Dutch Heart Foundation.

1 Popp RL, Schroeder JS, Stinson EB, et al. Ultrasonic studies for the early detection of acute cardiac rejection. Transplantation 1971;11:543-50.

2 Sagar KB, Hastillo A, Wolfgang TC, et al. Left ventricula mass by $M$-mode echocardiography in cardiac transplan patients with acute rejection. Circulation 1981; 64(suppl 2):216-20.

3 Paulsen W, Magid N, Sagar K, et al. Left ventricular function of heart allografts during acute rejection: an echocardiographic assessment. Heart Transplantation 1985;4:525-9.

4 Reid CJ, Yacoub MH. Determinants of left ventricular function one year after cardiac transplantation. Br Heart 1988;59:397-402.

5 Billingham ME. The diagnosis of acute cardiac rejection by endomyocardial biopsy. Bibl Cardiol 1988;43:83-102.

6 Angermann CE, Spes CH, Hart RJ, Kemkes B, Gokel M Theisen K. Echokardiograpische Diagnose akuter Abstosungsreaktionen bei herztransplantierten Patiente unter Cyclosporintherapie. Z Kardiol 1989;78:243-52 (English abstract).

7 Ciliberto GR, Cataldo G, Cipriani M, et al. Echocardiographic assessment of cardiac allograft rejection. Eur Heart $J$ 1989;10:400-8

8 Desruennes M, Corcos T, Cabrol A, et al. Doppler echocardiography for the diagnosis of acute cardiac allograft rejection. J Am Coll Cardiol 1988;12:63-70.

9 Dawkins KD, Oldershaw PJ, Billingham ME, et al Noninvasive assessment of cardiac allograft rejection. Transplant Proc 1985;17:215-7.

10 Mckoy RC, Uretsky BF, Kormos R, et al. Left ventricula hypertrophy in cyclosporine-induced systemic hypertenhypertrophy in cyclosporine-induced systemic hyperten-
sion after heart transplantation. Am J Cardiol 1988; 62:1140-2

11 Billingham ME. Endomyocardial biopsy interpretation in cyclosporine-treated cardiac recipients. Cardiac surgery: State of the art reviews. 1988;2:641-6.
12 St John Sutton MG, Reichek N, Kastor JA, et al. Computerized M-mode echocardiographic analysis of left ventricular dysfunction in cardiac amyloid. Circulation 1982;4:790-9.

13 Vine W, Flye MW, Jatlow P. Relationships of cyclosporine concentrations in serum, whole blood, and bile after renal and hepatic transplantation. Clin Chem 1986;32:1828-31.

14 Suffredini AF, Fromm RE, Parker MM, et al. The cardiovascular response of normal humans to the administration of cular response of normal humans to the adm

15 Gardin JM, Henry WL, Savage DD, Ware JH, Burn C Borer JS. Echocardiographic measurements in normal subjects: evaluation of an adult population without clinically apparent heart disease. J Clin Ultrasound 1979;7:439.

16 Kitabatake A, Inoue $M$, Asao M, et al. Transmitral bloodflow reflecting diastolic behaviour of the left ventricle in health and disease. Jpn Circulation J 1982;46:92-102.

17 Morgan JM, Raposo L, Clague JC, et al. Restrictive cardiomyoapthy and constrictive pericarditis: noninvasive distinction by digitised $M$ mode echocardiography. Br Heart J 1989;61:29-37.

18 Hausdorf G, Banner NS, Mitchell A, Khagani A, Martin M, Yacoub $M$. Diastolic function after cardiac and heart-lung transplantation. Br Heart J 1989;62:123-32.

19 Young JB, Leon CA, Short HD, et al. Evolution of hemodynamics after orthotopic heart and heart-lung transplantation: early restrictive patterns persisting in occult fashion. J Heart Transplant 1987;6:34-43.

20 St Goar FG Gibbons R, Schnittger I, Valantine HA, Popp RL. Left ventricular diastolic function. Doppler echocardiographic changes soon after cardiac transplantation Circulation 1990;82:872-8.

21 Brutsaert D, Rademakers F, Sys S, et al. Triple control of relaxation: implications in cardiac disease. Circulation 1984;69:190-6.

22 Spiegelhalter D, Stovin P. An analysis of repeated biopsies following cardiac transplantation. Stat Med 1983;2:33-40.

23 Valantine HA, Appleton CP, Hatle LK, Hunt SA, Stinson EB, Popp RL. Influence of recipient atrial contraction on left ventricular filling dynamics of the transplanted hear assessed by Doppler echocardiography. Am J Cardiol 1987;59:1159-63.

24 Appleton CP, Hatle LK, Popp RL. Heart tamponade and pericardial effusion: respiratory variation in transvulvar pericardial effusion: respiratory variation in transvulvar
flow velocities studied by Doppler echocardiography. $J$ Am Coll Cardiol 1988;11:1020-30.

25 Feneley M Kearny I, Farnsworth A, et al Mechanisms of the development and resolution of paradoxical interventricular septal motion after uncomplicated cardiac surgery. Am Heart J 1987;114:106-14. 